\title{
Lexicalized Hidden Markov Models for Part-of-Speech Tagging
}

\author{
Sang-Zoo Lee and Jun-ichi Tsujii \\ Department of Information Science \\ Graduate School of Science \\ University of Tokyo, Hongo 7-3-1. \\ Bunkyo-ku, 'Tokyo 113, Jajan \\ \{lec,tisujii\}(@is.s.u-tokyo.ac.jp)
}

\author{
Hae-Chang Rim \\ Department of Computer Science \\ Korca University \\ 1. 5-Ga Anam-Dong, Seongbuk-Gu \\ Scoul 136-701, Korea \\ rim(@)nlp.korea.ac.kr
}

\begin{abstract}
Since most previous works for HMM-based tagging consider only part-of-speech information in contexts, their models cannot utilizo lexical information which is crucial for resolving some morphological ambiguity. In this paper we introduce uniformly lexicalized JMMMs for partof-spech tagging in both English and Korcan. The lexicalized models use a simplified back-off smoothing technicue to overcome data sparseness. In experiments, lexiealized models achicve higher accuracy than non-lexicalized models and the back-off smoothing method mitigates data sparscness better than simple smoothing methods.
\end{abstract}

\section{Introduction}

Part-of-speech(POS) tagging is a process in which a proper loS tag is assigned to each word in raw texts. Wven though norphologically antbignous words have more than one POS tag, they belong to just one tag in a context. To resolve such ambiguity, taggers have to consult various sources of information such as lexical preferences (c.g. without consulting context, table is more probably a nom than a verb or: au adjective), tag n-gram contexts (e.g. after a non-possessive pronoum, table is more probably a verb than a noun or an adjective, as in they table an amendment), word 11-gram contexts (e.g. before lamp, table is more probably an adjective than a nom or a verb, as in I need a table lamp), and so on(Lee et al., 1999).

However, most previous HMM-based taggers consider only POS information in contexts, and so they cannot capture lexical information which is necessary for resolving some morphological ambiguity. Some recent. works have reported that tagging accuracy could be improved by using lexical information in their models such as the transformation-based patch rules(Brill, 1994), the maximum entropy model(liatnaparkhi, 1996), the statistical lexical rules(Lee et al., 1999), the IIMM considcring multi-words(Kim, 1996), the selectively lexicalized HMM(Kim ot al., 1999), and so on. In the previous works(Kim, 1996)(Kim et al., 1999), however, their HMMs were lexicalized selectively and restrictively.

In this paper we propose a method of miformly lexicalizing the standard HMM for partof-speech tagging in both English and Korcan. Becanse the sparse-data problem is more serious in lexicalized models than in the standard model, a simplified version of the woll-known back-off smoothing mothod is used to overcome the problem. For experiments, the Brown corpus(Francis, 1982) is used for English tagging and the KUNIP corpus(Lee et al., 1999) is used for Korean tagging. The experimental results show that lexicalized models perform better than non-lexicalized models and the simplified back-off smoothing technique can mitigate data sparseness better than simple smoothing techniques.

\section{The "standard" HMM}

We basically follow the notation of (Charniak et al., 1993) to describe Bayesian models. In this paper, we assume that $\left\{w^{1}, w^{2}, \ldots, w^{\omega}\right\}$ is a set of words, $\left\{t^{1}, t^{2}, \ldots, t^{\tau}\right\}$ is a set of POS tags, a sequence of random variables $W_{1, n}=$ $W_{1} W_{2} \ldots W_{n}$ is a sentence of $n$ words, and a sequence of random variables $T_{1, n}=T_{1} T_{2} \ldots T_{n}$ is a sequence of $n$ POS tags. Because each of random variables $W$ can take as its value any of the words in the vocabulary, we denote the value of $W_{i}$ by $w_{i}$ and a particular sequence of values for $W_{i, j}(i \leq j)$ by $w_{i, j}$. In a similar way, we denote the value of $T_{i}$ by $t_{i}$ and a particular 
sequence of values for $T_{i, j}(i \leq j)$ by $t_{i, j}$. For generality, terms $w_{i, j}$ and $t_{i, j}(i>j)$ are defined as being empty.

The purpose of Bayesian models for POS tagging is to find the most likely sequence of POS tags for a given sequence of words, as follows:

$T\left(w_{1, n}\right)=\underset{t_{1, n}}{\operatorname{argmax}} \operatorname{Pr}\left(T_{1, n}=t_{1, n} \mid W_{1, n}=w_{1, n}\right)$

Because reference to the random variables themselves can be omitted, the above equation becomes:

$$
T\left(w_{1, n}\right)=\underset{t_{1, n}}{\operatorname{argmax}} \operatorname{Pr}\left(t_{1, n} \mid w_{1, n}\right)
$$

Now, Eqn. 1 is transformed into Eqn. 2 since $\operatorname{Pr}\left(w_{1, n}\right)$ is constant for all $t_{1, n}$.

$$
\begin{aligned}
T\left(w_{1, n}\right) & =\underset{t_{1, n}}{\operatorname{argmax}} \frac{\operatorname{Pr}\left(t_{1, n}, w_{1, n}\right)}{\operatorname{Pr}\left(w_{1, n}\right)} \\
& =\underset{t_{1, n}}{\operatorname{argmax}} \operatorname{Pr}\left(t_{1, n}, w_{1, n}\right)
\end{aligned}
$$

Then, the probability $\operatorname{Pr}\left(t_{1, n}, w_{1, n}\right)$ is broken down into Eqn. 3 by using the chain rule.

$$
\operatorname{Pr}\left(t_{1, n}, w_{1, n}\right)=\prod_{i=1}^{n}\left(\begin{array}{c}
\operatorname{Pr}\left(t_{i} \mid t_{1, i-1}, w_{1, i-1}\right) \\
\times \operatorname{Pr}\left(w_{i} \mid t_{1, i}, w_{1, i-1}\right)
\end{array}\right)
$$

Because it is difficult to compute Eqn. 3, the standard HMM simplified it by making a strict Markov assumption to get a more tractable form.

$$
\operatorname{Pr}\left(t_{1, n}, w_{1, n}\right) \approx \prod_{i=1}^{n}\left(\begin{array}{l}
\operatorname{Pr}\left(t_{i} \mid t_{i-K, i-1}\right) \\
\times \operatorname{Pr}\left(w_{i} \mid t_{i}\right)
\end{array}\right)
$$

In the standard HMM, the probability of the current tag $t_{i}$ depends on only the previous $K$ tags $t_{i-K, i-1}$ and the probability of the current word $w_{i}$ depends on only the current tag ${ }^{1}$. Therefore, this model cannot consider lexical information in contexts.

\section{Lexicalized HMMs}

In English POS tagging, the tagging unit is a word. On the contrary, Korcan POS tagging prefers a morpheme ${ }^{2}$.

\footnotetext{
${ }^{1}$ Usually, $K$ is determined as 1 (bigram as in (Charniak et al., 1993)) or 2 (trigram as in (Merialdo, 1991)).

${ }^{2}$ The main reason is that the number of word-unit tags is not finite because Korean words can be freely and newly formed by agglutinating morphemes(Lee et al., 1999).
}

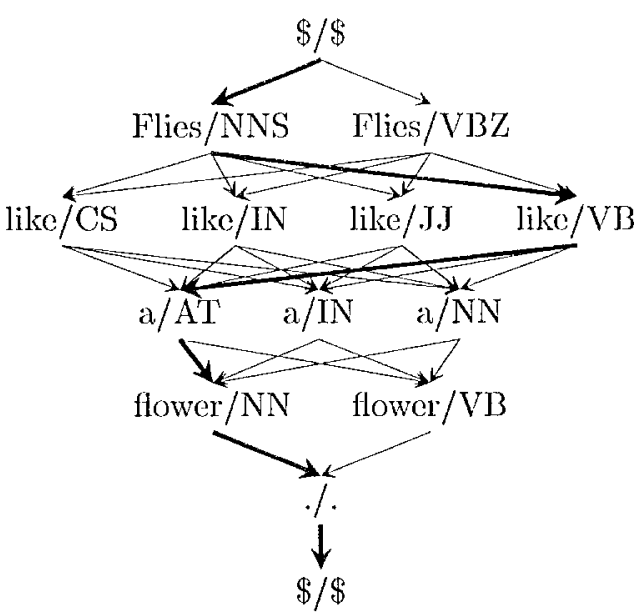

Figure 1: A word-unit lattice of "Flies like a flower ."

Figure 1 shows a word-unit lattice of an English sentence, "Flies like a flower.", where each node has a word and its word-mit tag. Figure 2 shows a morpheme-unit lattice of a Korean sentence, "NeoNeun Hal Su issDa.", where each node has a morpheme and its morphemeunit tag. In case of Korcan, transitions across a word boundary, which are depicted by a solid line, are distinguished from transitions within a word, which are depicted by a dotted line. In both cases, sequences connected by bold lines indicate the most likely sequences.

\subsection{Word-unit models}

Lexicalized HMMs for word-unit tagging are defined by making a less strict Markov assumption, as follows:

$$
\begin{aligned}
& \Lambda\left(T_{(K, J)}, W_{(L, I)}\right) \vDash \operatorname{Pr}\left(t_{1, n}, w_{1, n}\right) \\
& \approx \prod_{i=1}^{n}\left(\begin{array}{c}
\operatorname{Pr}\left(t_{i} \mid t_{i-K, i-1}, w_{i-J, i-1}\right) \\
\times \operatorname{Pr}\left(w_{i} \mid t_{i-L, i}, w_{i-I, i-1}\right)
\end{array}\right)
\end{aligned}
$$

In models $\Lambda\left(T_{(K, J)}, W_{(L, I)}\right)$, the probability of the current $\operatorname{tag} t_{i}$ depends on both the previous $K$ tags $t_{i-K, i-1}$ and the previous $J$ words $w_{i-J, i-1}$ and the probability of the current word $w_{i}$ depends on the current tag and the previous $L$ tags $t_{i-L, i}$ and the previous $I$ words $w_{i-I, i-1}$. So, they can consider lexical information. In experiments, we set $K$ as 1 or $2, J$ as 0 or $K, L$ as 1 or 2 , and $I$ as 0 or $L$. If $J$ and $I$ are zero, the above models are non-lexicalized models. Otherwise, they are lexicalized models. 


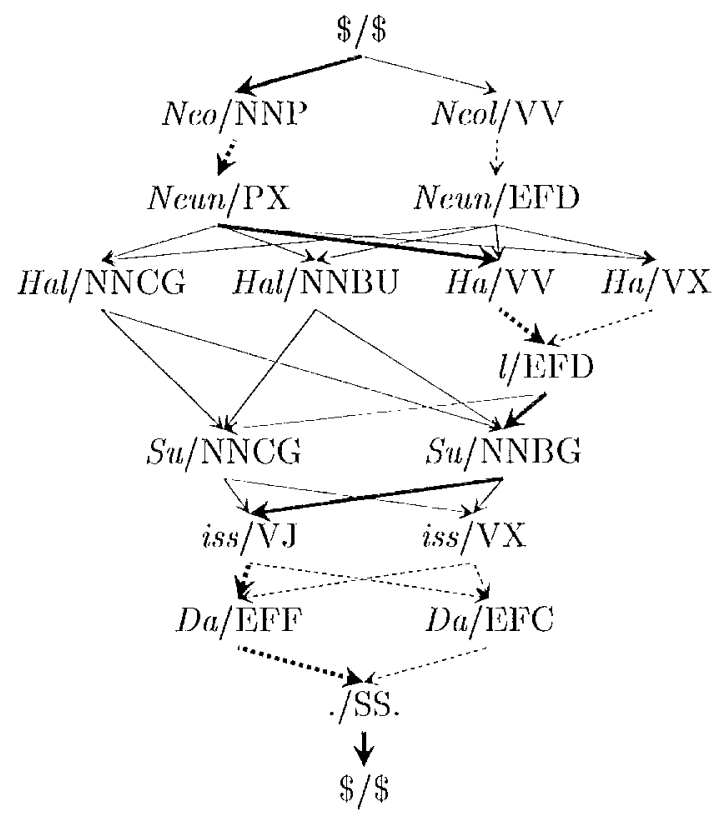

ligure 2: A morpheme-unit latice of "NeoNeun Hal Su issDa." (= You can do it.)

In a lexicalized model $\Lambda\left(T_{(2,2)}^{\prime}, W_{(2,2)}\right)$, for $\mathrm{cx}-$ ample, the probalility of a nock "a/AT" of the most likely sequence in Figure 1 is calculated as follows:

$$
\begin{array}{l|l}
\operatorname{Pr}(A T & N N S, V B, \text { Flies, like }) \\
\times \operatorname{Pr}(a & A T, N N S, V I 3, \text { Iflices, like })
\end{array}
$$

\subsection{Morpheme-unit models}

Bayesian models for morpheme-mit tagging find the most likely secpuence of morphemes and corresponding tags for a given sequence of words, as follows:

$$
\begin{aligned}
T^{\prime}\left(w_{1, n}\right) & =\underset{c_{1, u}, m_{1, u}}{\operatorname{argmax}} \operatorname{Pr}\left(c_{1, u}, m_{1, u} \mid w_{1, u}\right) \\
& \approx \underset{c_{1, u}, m_{1, u}}{\operatorname{argmax}} \operatorname{Pr}\left(c_{1, u}, p_{2, u}, m_{1, u}\right)
\end{aligned}
$$

In the above equations, $u(\geq n)$ denotes the number of morphemes in a sequence corresponding the given word sequence, $c$ denotes a morpheme-1nnit tag, $m$ denotes a morpheme, and $p$ denotes a type of transition from the previous tag to the current tag. $p$ can have one of two values, "\#" denoting a transition across a word boundary and " + " denoting a transition within a word. Because it is difficult to calculate Eqn. 6, the word sequence term $w_{1, n}$ is usually ignored as in Eqn. 7. Instead, we introduce $p$ in
Equn. 7 t,o consider word-spacing ${ }^{3}$.

The probability $\operatorname{Pr}\left(c_{1, u}, p_{2, u}, m_{1, u}\right)$ is also broken down into Eqn. 8 by using the chain rule.

$$
\begin{aligned}
& \operatorname{Pr}\left(c_{1, u}, p_{2, u}, m_{1, u}\right) \\
& =\prod_{i=1}^{u}\left(\begin{array}{c}
\operatorname{Pr}\left(c_{i}, p_{i} \mid c_{1, i-1}, p_{2, i-1}, m_{1, i-1}\right) \\
\times \operatorname{Pr}\left(m_{i} \mid c_{1, i}, p_{2, i}, m_{1, i-1}\right)
\end{array}\right)
\end{aligned}
$$

Bocause Ecpl. 8 is not casy to compute, it is simplified by making a Markov assumption to get a more tractable form.

In a similar way to the case of word-mit tagging, lexicalized HMMs for morpheme-unit tagging are defined by making a less strict Markov assumption, as follows:

$$
\begin{aligned}
& \Lambda\left(C_{[s](K, J)}, M_{[s](I, I)}\right) \models \operatorname{Pr}\left(c_{1, u}, p_{2, u}, m_{1, u}\right) \\
& \approx \prod_{i=1}^{u} \operatorname{Pr}\left(c_{i}\left[, p_{i}\right] \mid \begin{array}{l}
c_{i-K, i-1}\left[, p_{i-K+1, i-1}\right], \\
m_{i-J, i-1}
\end{array}\right)
\end{aligned}
$$

In models $\Lambda\left(C_{[s](K, I)}, M_{[s](L, I)}\right)$, the probability of the current morpheme tag $c_{i}$ depends on both the previous $K$ tags $c_{i-K, i-1}$ (optionally, the types of their transition $\left.p_{i-K+1, i-1}\right)$ and the previous $f$ morphemes $m_{i-J, i-1}$ and the probability of the current morpheme $m_{i}$ depends on the current tage and the previous $L$ tagss $c_{i-l, i}$ (optionally, the types of their transition $p i,-\ldots+1, i)$ and the previous $I$ morphemes $m_{i-1, i-1}$. So, they cann also consider lexical information.

In a lexicalized model $\Lambda\left(C_{s(2,2)}, M_{(2,2)}\right)$ where word-spacing is considered only in the tag probabilitics, for example, the probability of a node, "Su/NNBG" of the most likely sequence in Figure 2 is calculated as follows:

$$
\begin{aligned}
& \operatorname{Pr}(N N B G, \# \mid V V, E F D,+, H a, l) \\
& \times \operatorname{Pr}(S u \mid V V, E F D, N N B G, H a, l)
\end{aligned}
$$

\subsection{Parameter estimation}

In supervised learning, the simpliest parameter estimation is the maximum likelihood(ML) estimation(Duda et al., 1973) which maximizes the probability of a training set. The ML estimate of tag $(K+1)$-gram probability, $\operatorname{Pr}_{M L}\left(t_{i} \mid\right.$ $\left.t_{i-K, i-1}\right)$, is calculated as follows:

$$
\operatorname{Pr}_{M L}\left(t_{i} \mid t_{i-K, i-1}\right)=\frac{\mathrm{Fq}\left(t_{i-K, i}\right)}{\mathrm{Fq}\left(t_{i-K, i-1}\right)}
$$

\footnotetext{
${ }^{3}$ Most previous HMM-based Korean taggers except (Kim ot al., 1998) did not consider word-spacing.
} 
where the function $\mathrm{Fq}(x)$ returns the frequency of $x$ in the training set. When using the maximum likelihood estimation, data sparseness is more serious in lexicalized models than in nonlexicalized models because the former has even more parameters than the latter.

In (Chen, 1996), where various smoothing techniques was tested for a language model by using the perplexity measure, a back-off smoothing(Katz, 1987) is said to perform better on a small traning set than other methods. In the back-off smoothing, the smoothed probability of tag $(K+1)$-gram $\operatorname{Pr}_{S B O}\left(t_{i} \mid t_{i-K, i-1}\right)$ is calculated as follows:

$$
\begin{aligned}
& \operatorname{Pr}_{S B O}\left(t_{i} \mid t_{i-K, i-1}\right)= \\
& \left\{\begin{array}{l}
d_{r} \operatorname{Pr}_{M L}\left(t_{i} \mid t_{i-K, i-1}\right) \quad \cdot \quad \text { if } r>0 \\
\alpha\left(t_{i-K, i-1}\right) \operatorname{Pr}_{S B O}\left(t_{i} \mid t_{i-K+1, i-1}\right) \text { if } r=0
\end{array}\right. \\
& \text { where } r=\mathrm{Fq}\left(t_{i-K, i}\right), r^{*}=(r+1) \frac{n_{r+1}}{n_{r}} \\
& d_{r}=\frac{\frac{r^{*}}{r}-\frac{(r+1) \times n_{r+1}}{n_{1}}}{1-\frac{(r+1) \times n_{r+1}}{n_{1}}}
\end{aligned}
$$

$n_{r}$ denotes the number of $(K+1)$-gram whose frequency is $r$, and the coefficient $d_{r}$ is called the discount ratio, which reflects the GoodTuring estimate(Good, 1953) ${ }^{4}$. Eqn. 11 means that $\operatorname{Pr}_{S B O}\left(t_{i} \mid t_{i-K, i-1}\right)$ is under-etimated by $d_{r}$ than its maximum likelihood estimate, if $r>0$, or is backed off by its smoothing torm $\operatorname{Pr}_{S B O}\left(t_{i} \mid t_{i-K+1, i-1}\right)$ in proportion to the value of the function $\alpha\left(t_{i-K, i-1}\right)$ of its conditional term $t_{i-K, i-1}$, if $r=0$.

However, because Eqn. 11 requires complicated computation in $\alpha\left(t_{i-K, i-1}\right)$, we simplify it to get a function of the frequency of a conditional term, as follows:

$$
\begin{aligned}
& \alpha\left(\mathrm{Fq}\left(t_{i-K, i-1}\right)=f\right)= \\
& \quad \Delta \times \frac{\mathrm{E}\left[\mathrm{Fq}\left(t_{i-K, i-1}\right)=f\right]}{\sum_{f=0}^{\infty} \mathrm{E}\left[\mathrm{Fq}\left(t_{i-K, i-1}\right)=f\right]}
\end{aligned}
$$

where $\Delta=1-\sum_{t_{i-K, i}, r>0} \operatorname{Pr}_{S B O}\left(t_{i} \mid t_{i-K, i-1}\right)$,

$$
\sum_{t_{i-K+1, i}, r=0, F q\left(t_{i-K, i-1}\right)=f}^{\mathrm{E}\left[\mathrm{Fq}\left(t_{i-K, i-1}\right)=f\right]=} \operatorname{Pr}_{S B O}\left(t_{i} \mid t_{i-K+1, i-1}\right)
$$

In Eqn. 12, the range of $f$ is bucketed into 7

\footnotetext{
${ }^{4}$ Katz said that $d_{r}=1$ if $r>5$.
}

regions such as $f=0,1,2,3,4,5$ and $f \geq 6$ since it is also difficult to compute this equation for all possible values of $f$.

Using the formalism of our simplified back-off smoothing, each of probabilities whose ML estimate is zero is backed off by its corresponding smoothing term. In experiments, the smoothing terms of $\operatorname{Pr}_{S B O}\left(t_{i} \mid t_{i-K, i-1}, w_{i-J, i-1}\right)$ are determined as follows:

$$
\begin{cases}\operatorname{Pr}_{S B O}\left(t_{i} \mid \begin{array}{c}
t_{i-K+1, i-1}, \\
w_{i-J+1, i-1}
\end{array}\right) & \text { if } K \geq 1, J>1 \\
\operatorname{Pr}_{S B O}\left(t_{i} \mid t_{i-K, i-1}\right) & \text { if } K \geq 1, J=1 \\
\operatorname{Pr}_{S B O}\left(t_{i} \mid t_{i-K+1, i-1}\right) & \text { if } K \geq 1, J=0 \\
\operatorname{Pr}_{A D}\left(t_{i}\right) & \text { if } K=0, J=0\end{cases}
$$

Also, the smoothing terms of $\operatorname{Pr}_{S B O}\left(w_{i}\right.$ $\left.t_{i-L, i}, w_{i-l, i-1}\right)$ are determined as follows:

$$
\begin{cases}\operatorname{Pr}_{S B O}\left(w_{i} \mid t_{i-L+1, i},\right. & \text { if } L \geq 1, I>1 \\ \operatorname{Pr}_{S B O}\left(w_{i} \mid t_{i-L, i, i-1}\right) & \text { if } L \geq 1, I=1 \\ \operatorname{Pr}_{S B O}\left(w_{i} \mid t_{i-L+1, i}\right) & \text { if } L \geq 1, I=0 \\ \operatorname{Pr}_{S B O}\left(w_{i}\right) & \text { if } L=0, I=0 \\ \operatorname{Pr}_{A D}\left(w_{i}\right) & \text { if } L=-1, I=0\end{cases}
$$

In Eqn. 13 and 14, the smoothing term of a unigram probability is calculated by using an additive smoothing with $\delta=10^{-2}$ which is chosen through experiments. The equation for the additive smoothing(Chen, 1996) is as follows:

$$
\underset{A D}{\operatorname{Pr}}\left(t_{i} \mid t_{i-K, i-1}\right)=\frac{\mathrm{Fq}\left(t_{i-K, i}\right)+\delta}{\sum_{t_{i}}\left(\mathrm{Fq}\left(t_{i-K, i}\right)+\delta\right)}
$$

In a similar way, the smoothing terms of paramcters in Eqn. 9 are determined.

\subsection{Model decoding}

From the viewpoint of the lattice structure, the problem of POS tagging can be regarded as the problem of finding the most likely path from the start node $(\$ / \$)$ to the end node $(\$ / \$)$. The Viterbi search algorithm(Forney, 1973), which has been used for HMM decoding, can be effectively applied to this task just with slight modification $^{5}$.

\section{Experiments}

\subsection{Environment}

In experiments, the Brown corpus is used for English POS tagging and the KUNLP corpus

\footnotetext{
${ }^{5}$ Such modification is explained in detail in (Lee, 1999).
} 


\begin{tabular}{|l||r|r|}
\hline & Brown & KUNIIP \\
\hline \hline NW & $1,113,189$ & 167,115 \\
\hline NS & 53,885 & 15,211 \\
\hline NT & 82 & 65 \\
\hline DA & 1.64 & 3.41 \\
\hline RUA & $61.54 \%$ & $26.72 \%$ \\
\hline
\end{tabular}

NW Number of words. NS Number of sentences. NT Number of tags (morpheme-nnit tag for KUNLP). DA Degree of ambiguity (i.c. the number of tags per word). RUA Ratio of unambiguous words.

Table 1: Information about the Brown corpus and the KUNLP corpus

\begin{tabular}{|l||c|c|}
\hline & Inside-test & Outside-test \\
\hline$\overline{\mathrm{ML}}$ & 95.57 & $\mathbf{9 4 . 9 7}$ \\
\hline$\overline{\mathrm{AD}(\delta=1)}$ & 93.92 & 93.02 \\
\hline$\overline{\mathrm{AD}}\left(\delta=10^{-1}\right)$ & 95.02 & 94.79 \\
\hline $\mathrm{AD}\left(\delta=10^{-2}\right)$ & 95.42 & $\mathbf{9 5 . 0 8}$ \\
\hline $\mathrm{AD}\left(\delta=10^{-3}\right)$ & 95.55 & 95.05 \\
\hline $\mathrm{AD}\left(\delta=10^{-4}\right)$ & 95.57 & 94.98 \\
\hline $\mathrm{AD}\left(\delta=10^{-5}\right)$ & 95.57 & 94.94 \\
\hline $\mathrm{AD}\left(\delta=10^{-6}\right)$ & 95.57 & 94.91 \\
\hline $\mathrm{AD}\left(\delta=10^{-7}\right)$ & 95.57 & 94.89 \\
\hline $\mathrm{AD}\left(\delta=10^{-8}\right)$ & 95.57 & 94.87 \\
\hline $\mathrm{SBO}$ & 95.55 & $\mathbf{9 5 . 2 5}$ \\
\hline
\end{tabular}

ML Maximum likelihood cstimate (with simple smoothing). AD Additive smoothing. S13O Simplified back-off smoothing.

Table 2: 'Tagging accuracy of $\Lambda\left(C_{(1: 0)}, M_{(0: 0)}\right)$

for Korcan POS tagging. Table 1 shows somo information about both corpora ${ }^{6}$. Wach of them was segmented into two parts, the training set of $90 \%$ and the test set of $10 \%$, in the way that each sentence in the test set was extracted from every 10 sentence. According to Table 1, Korean is saicl to be more difficult to disambiguate than English.

We assume "closed" vocabulary for: English and "open" vocabulary for Korean since we do not have any English morphological analyzer consistent with the Brown corpus. Therefore, for morphological analysis of English, we just

\footnotetext{
${ }^{6}$ Note that some sentences, which have composite tags(such as "IIV+TO" in "hafta"), "ILLEEGAL" tag, or. "NIL" tag, were removed from the Brown corpus and tags with "**" (not) such as "J3EZ*" were replaced by corresponding tags without "*" such as "BEZ".
}
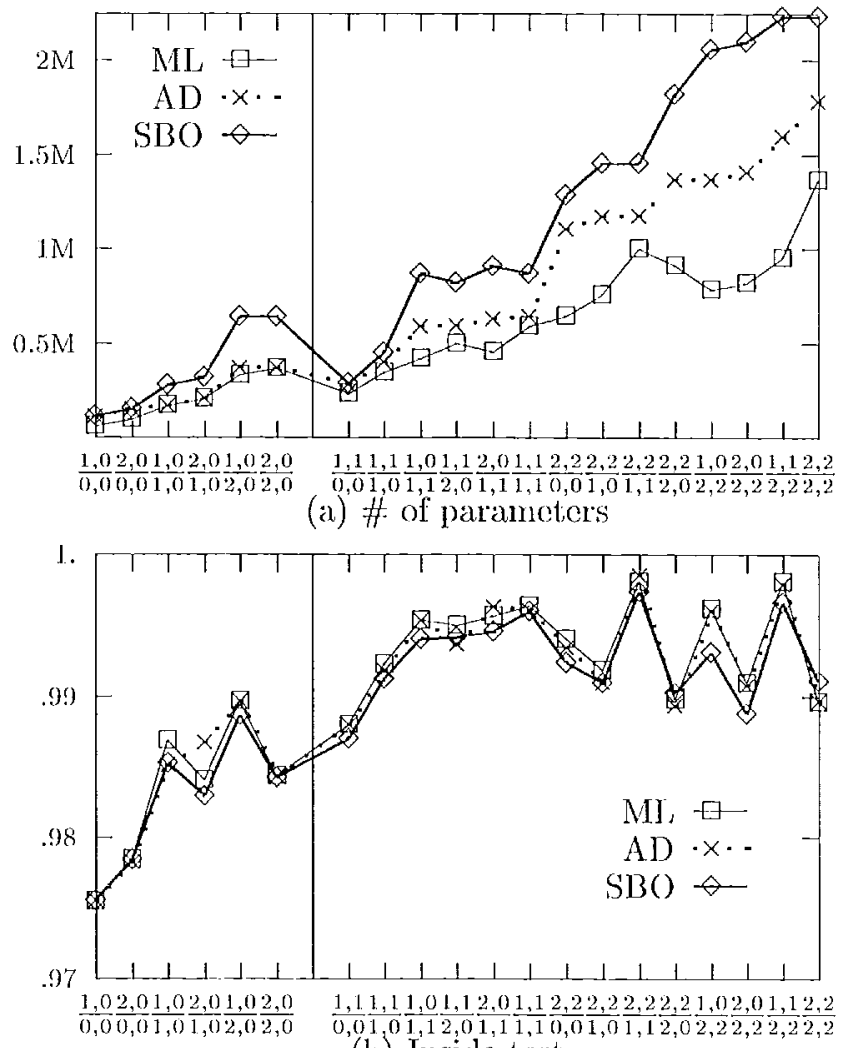

(b) Inside-test
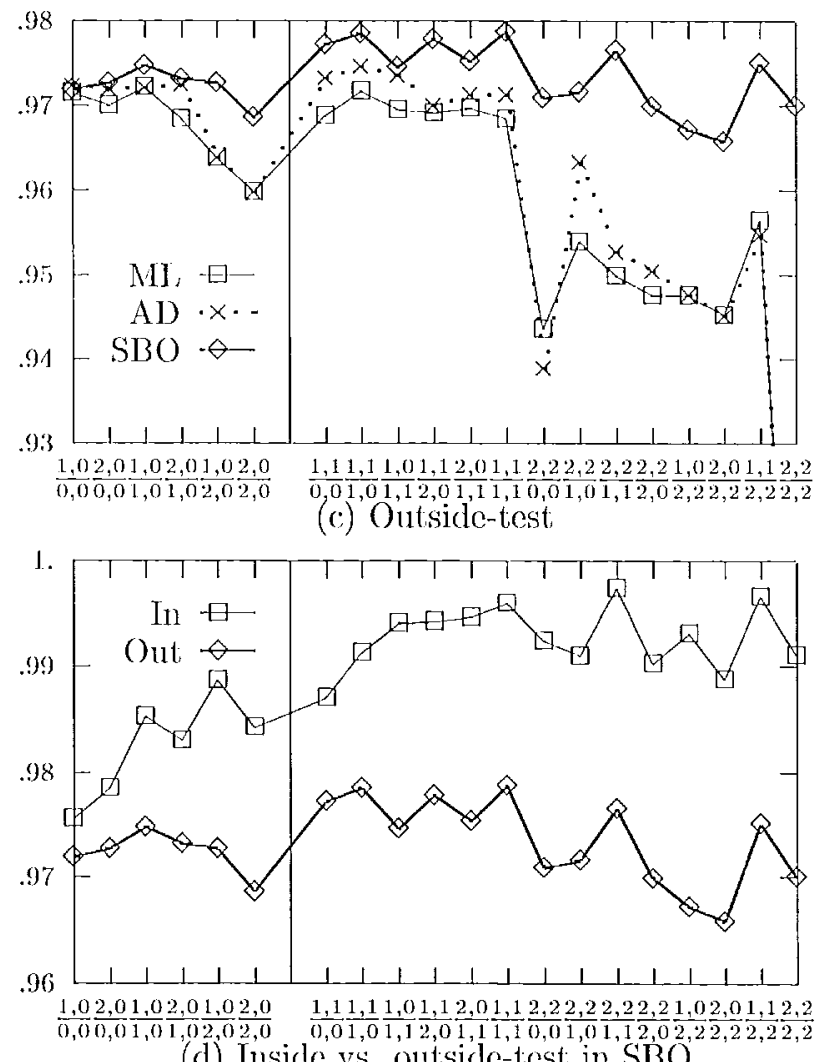

Figure 3: Results of English tagging 
looked up the dictionary tailored to the Brown corpus. In case of Korean, we have used a Korean morphological analyzer(Lee, 1999) which is consistent with the KUNLP corpus.

\subsection{Results and evaluation}

Table 2 shows the tagging accuracy of the simplest HMM, $\Lambda\left(C_{(1: 0)}, M_{(0: 0)}\right)$, for Korcan tagging, according to various smoothing methods $^{7}$. Note that $M L$ denotes a simple smoothing method where ML estimates with probability less than $10^{-9}$ arc smoothed and replaced by $10^{-9}$. Because, in the outside-test, $A D\left(\delta=10^{-2}\right)$ performs better than $M L$ and $A D\left(\delta \neq 10^{-2}\right)$, we use $\delta=10^{-2}$ in our additive smoothing. According to Table 2, SBO performs well even in the simplest HMM.

Figure 3 illustrates 4 graphs about the results of English tagging: (a) the number of parameters in each model, (b) the accuracy of each model for the training set, (c) the accuracy of each model for the test set, and (d) the accuracy of each model with $S B O$ for both training and test set. Here, labels in $\mathrm{x}$-axis specify models in the way that $\frac{K, I}{I, I}$ denotes $\Lambda\left(T_{(K, J)}, W_{(I, I)}\right)$. Therefore, the first 6 models are non-lexicalized models and the others are lexicalized models.

Actually, SBO uses more parametess than others. The three smoothing methods, $M L$, $A D, S B O$, perform well for the training set since the inside-tests usually have little data sparseness. On the other hand, for the unseen test set, the simple methods, $M L$ and $A D$, cannot mitigate the data sparseness problem, especially in sophisticated models. However, our method $S B O$ can overcome the problem, as shown in Figure 3(c). Also, we can see in Figure 3(d) that some lexicalized modcls achieve higher accuracy than non-lexicalized models. We can say that the best lexicalized model, $\Lambda\left(T_{(1,1)}, W_{(1,1)}\right)$ using $S B O$, improved the simple bigram model, $\Lambda\left(T_{(1,0)}, W_{(0,0)}\right)$ using $S B O$, from $97.19 \%$ to $97.87 \%$ (the error reduction ratio of $24.20 \%)$. Interestingly, some lexicalized models (such as $\Lambda\left(T_{(1,1)}, W_{(0,0)}\right)$ and $\left.\Lambda\left(T_{(1,1)}, W_{(1,0)}\right)\right)$, which have a relatively small number of parameters, perform better than non-lexicalized models in the case of outsidetests using SBO. Unfortunately, we cannot $\mathrm{ex}$ -

\footnotetext{
${ }^{7}$ Inside-test means an experiment on the training set itself and outside-test an experiment on the test set.
}

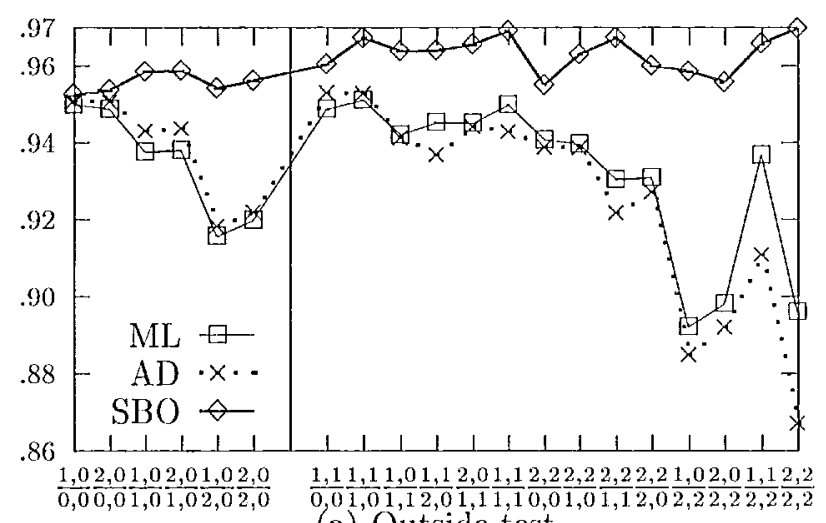

(a) Outside-test

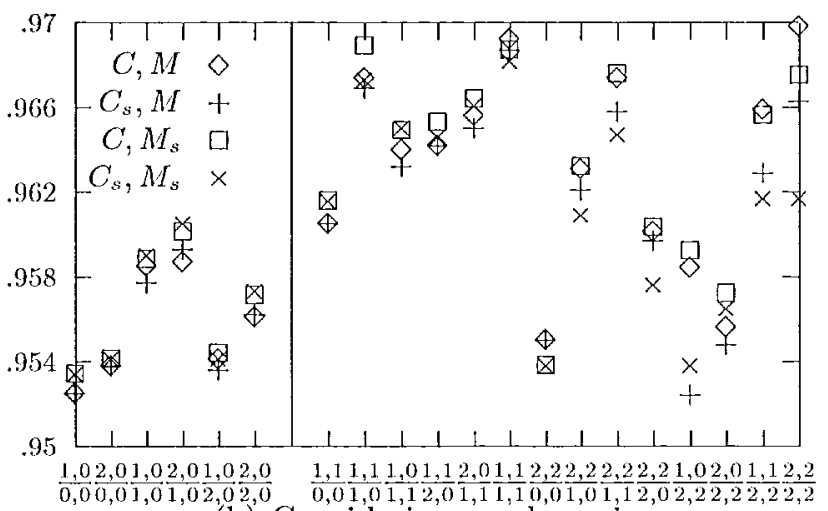

(b) Considering word-spacing

Figure 4: Results of Korean tagging

pect the result of outside-tests from that of inside-tests because there is no direct relation between them.

Figure 4 includes 2 graphs about the results of Korcan tagging: (a) the outside accuracy of each model $\Lambda\left(C_{(K, J)}, M_{(L, I)}\right)$ and (b) the outside accuracy of each model $\Lambda\left(C_{[s](K, J)}, M_{[s](I, I)}\right)$ with/without considering word-spacing when using $S B O$. Here, labels in $\mathrm{x}$-axis specify models in the way that $\frac{K, J}{L, I}$ denotes $\Lambda\left(C_{[s](K, J)}, M_{[s](I, I)}\right)$ and, for example, $C_{s}, M$ in (b) denotes $\Lambda\left(C_{s(K, J)}, M_{(L, I)}\right)$.

As shown in Figure 4, the simple mothods, $M L$ and $A D$, cannot mitigate that sparsedata problem, but our method SBO can overcome it. Also, some lexicalized models perform better than non-lexicalized models. On the other hand, considering word-spacing gives good clues to the models sometimes, but yet we cannot say what is the best way. From the experimental results, we can say that the best model, $\Lambda\left(C_{(2,2)}, M_{(2,2)}\right)$ using $S B O$, improved the previous models, $\Lambda\left(C_{(1,0)}, M_{(0,0)}\right)$ us- 
ing $M L\left(\right.$ Lec, 1995), and $\Lambda\left(C_{s(1,0)}, M_{(0,0)}\right)$ using $M L($ Kim et al., 1998), from $94.97 \%$ and $95.05 \%$ to $96.98 \%$ (the crror reduction ratio of $39.95 \%$ and $38.99 \%$ ) respectively.

\section{Conclusion}

We have presented uniformly lexicalized HMMs for POS tagging of English and Korcan. In the models, data sparseness was effectively mitigated by using our simplified back-off smoothing. From the experiments, we have observed that lexical information is useful for POS tagging in IIMMs, as is in other models, and our lexicalized models improved non-lexicalized models by the error reduction ratio of $24.20 \%$ (in English tagging) and 39.95\% (in Korcan tagging).

Generally, the miform extension of models recuires rapid increase of parameters, and hence suffers from large storage and sparse data. Recently in many areas where HMMs are used, many eflorts to extend models non-uniformly have been made, sometimes resulting in noticeable improvement. For this reason, we are trying to transform our uniform models into nonuniform models, which may be more effective in terms of both space complexity and reliable estimation of paremeters, without loss of accuracy.

\section{References}

E. Brill. 1994. Some Advances in. Transformation-Based Part of Speech Tagging. In Proc. of the 19th Nat'l Conf. on Artificial Intelligence(AAAI-94), 722-727.

E. Charniak, C. Hendrickson, N. Jacobson, and M. Perkowitz. 1993. Equations for Partof-Specch Tagging. In Proce of the 11th Nat'l Conf. on Artificial Intelligence(AAAI93), 784-789.

S. F. Chen. 1996. Building Probabilistic Models for Natural Language. Doctoral Dissertation, Harvard University, USA.

R. O. Duda and R. E. Hart. 1973. Pattern Classification and Scene Analysis. John Wiley.

G. D. Forney. 1973. The Viterbi Algorithm. In Proc. of the IEEE, 61:268-278.

W. N. Francis and H. Kučcra. 1982. Frequency Analysis of English Usage: Lexicon and Grammar. Houghton Mifflin Company, Boston, Massachusetts.
I. J. Good. 1953. "The Population Frequencies of Species and the Estimation of Population Parameters," In Biometrika, 40(34):237-264.

S. M. Katz. 1987. Estimation of Probabilities from Sparse Data for the Language Model Component of a Speech Recognizer. In IEEE Transactions on Acoustics, Speech and Signal Processing(ASSP), 35(3):400-401.

J.-D. Kim, S.-Z. Lee, and H.-C. Rim. 1998. A Morpheme-Unit POS Tagging Model Considering Word-Spacing. In Proc. of the $10^{\text {th }}$ National Conference on Korean Information Processing, 3-8.

J.-D. Kim, S.-Z. Lee, and H.-C. Rim. 1999. HMM Specialization with Selective Lexicalization. In Proc. of the joint SIGDAT Conference on Empirical Methods in Natural Ianguage Processing and Very Large Corpora(EMNLP-VLC-99), 144-148.

J.-H. Kinn. 1996. Lexical Disambiguation with Error-Driven Learning. Doctoral Dissertation, Korea Advanced Institute of Science and Technology(KAIST), Korea.

S.-H. Lee. 1995. Korean POS Tagging System Considering Unknown Words. Master 'Thesis, Korea Advanced Institute of Science and Techmology(KAIST), Korea.

S.-Z. Lee, J.-I). Kim, W.-H. Ryu, and H.C. Rim. 1999. A Part-of-Spech Tagging Model Using Lexical Rules Based on Corpus Statistics. In Proc. of the International Conference on Computer Processing of Oriental Languages (ICCPOL-99), 385-390.

S.--Z. Lec. 1999. New Statistical Models for Automatic P'OS Tagging. Doctoral Dissertation, Korca University, Korea.

B. Merialdo. 1991. Tagging Text with a Probabilistic: Model. In Proc. of the International Conference on Acoustic, Specch and Signal Processing(ICASSP-91), 809-812.

A. Ratnaparkhi. 1996. A Maximum Entropy Model for Part-of-Speech Tagging. In Proc. of the Empirical Methods in Natural Language Processing Conference(EMNLP-96), 133-142. 